\title{
Scientific Report (2002-2004)
}

Studies of the Nonlinear Interactions between Optical-Mixing-Controlled Stimulated Scattering Instabilities in Laser-Produced Plasmas

DOE SSAA Grant \# DE-FG03-03NA00059 
The main goal of our research is to examine important nonlinear kinetic laser-plasma interaction phenomena not reducible to a fluid description adjusted by the addition of ad hoc frequency shifts and damping rates calibrated to fit some narrow parameter range experimental data set. We study the kinetic phenomena that permeate the scene of laser-plasma interactions, made more clear and discernible by the use of optical mixing techniques. Since these techniques bring out the details of coherent multiwave interactions being driven simultaneously by two coherent large amplitude laser beams, we can simulate and study coherent nonlinear phenomena in larger and more unstable systems, such as the NIF and LMJ, in greater detail than before.

We do this with Vlasov simulations, Omega experiments at LLE and by using the Trident laser at LANL. Our approach begins by systematic studies using Vlasov simulations of these multiple high frequency wave interaction processes, to design experiments and to conduct such experiments on Omega and Trident. We have been studying the interactions simultaneously driven by two different color laser beams to generate electron plasma waves (EPW) and KEEN (Kinetic Electrostatic Electron Nonlinear) waves in laser-produced plasmas. KEEN waves were discovered by our theoretical and computational efforts as part of this project. We expect to observe them experimentally in June 2004 on Trident at LANL.

In our latest experiments on Omega, we have discovered a new process whereby a blue beam amplifies a green beam via the generation of an EPW. The green beam car then Raman backscatter which in the absence of the blue beam was below threshold. In our upcoming campaign in Sept. 2004, we will probe this phenomenon further including polarization rotators, appropriately designed new phase plates and an attempt to generate EPWs and ion acoustic waves (IAW) simultaneously by two independent optical mixing processes.

This work has been done under the leadership of the Principle Investigator (PI), Dr. Bedros Afeyan. The simulations have been done at Polymath Research inc, (PRI) with Dr. Vlad Savchenko (a post doc.) and Kirk Won, modifying improving and running the Vlasov-Poisson and Vlasov-Maxwell codes with the help of Dr. Viktor Decyk of UCLA, especially in matters related to parallelization. Michel AlbrechtMarc, a physics graduate student at Univ. Henry Poincare', in Nancy, FR., has been working with us on blue-green EPW generation simulations as well. Our codes are 
used to study KEEN wave generation systematically as well as EPW and IAW generation processes with and without external noise introduced in the plasma model. A large suite of diagnostic tools has been created in order to ferret out the physics behind these complicated multi-wave processes.

The experiments on Omega are done with the participation of scientists from LLNL and LANL. In particular, David Montgomery of Los Alamos, Robert Kirkwood and Christoph Niemann (a post doc.) of Livermore and the PRI team which includes the PI, K. Won and Marine Mardirian have been responsible for the success of our campaign at LLE in 2003. This team together with the LANL post doc., John Kline, and the UC Berkeley physics department graduate student, Will Bersche will conduc1 our upvoming experiments on Omega in September of this year.

The KEEN wave detection Trident experiments in June of this year will involve Will Bersche of UC Berkeley, a graduate student in the physics department, David Montgomery and John Kline,(a post doc.) of LANL and the PRI team. This three week Trident campaign will be used to drive new (KEEN) waves in parameter regimes never before tried in any experiment to identify modes heretofore unknown to plasma physics, being in a spectral gap where no modes were thought to exist. This new fundamental plasma physics phenomenon is also tied to single species magnetized trap-plasma phenomena that is the central subject of Will Bersche's thesi at Berkeley. KEEN waves have significant implications to the entire edifice of phenomena thought to occur in LPI experiments. If these waves are ubiquitous and were thought not to exist, many of the interpretive details of LPI experiments past, present and future would have to be modified. 
MO3.4

We include two papers describing some of our research highlights.

\title{
Kinetic Electrostatic Electron Nonlinear (KEEN) Waves and their Interactions Driven by the Ponderomotive Force of Crossing Laser Beams
}

\author{
Bedros Afeyan, ${ }^{a}$ K. Won, ${ }^{a}$ V. Savchenko, ${ }^{a}$ T. W. Johnston, ${ }^{\mathrm{b}}$ A. Ghizzo, ${ }^{\mathrm{c}}$ P. \\ Bertrand $^{\mathrm{c}}$ \\ ${ }^{a}$ Polymath Research Inc., Pleasanton, CA \\ ${ }^{b}$ INRS, Varennes, Quebec, Canada \\ ${ }^{c}$ Universite Henri Poincare, Nancy, France
}

\begin{abstract}
We have found, using $1 D$ periodic Vlasov-Poisson simulations, new nonlinear, nonstationary, stable, long lived, coherent structures in phase space, called kinetic electrostatic electron nonlinear (KEEN) waves. Ponderomotively driven for a short period of time, at a particular frequency and wavenumber, well inside the band gap that was thought to exist between electron plasma and electron acoustic wave frequencies, KEEN waves are seen to selfconsistently form, and persist for thousands of plasma periods. KEEN waves are comprised of 4 or 5 significant phase-locked harmonic modes. They persist only when driven hard enough. They also merge when two or more at different frequencies are driven sequentially. However, the final stable KEEN state that emerges is highly sensitive to their relative order of excitation. KEEN waves also interact quite strongly with electron plasma waves (EPW) especially when their harmonics are close to being resonant with the EPW frequency. The common assumption that whenever sufficiently large amplitude coherent laser energy is present in an unmagnetized plasma, EPWs and IAWs are the only waves with which the electromagnetic energy can interact may require reconsideration.
\end{abstract}

Keywords: Kinetic electrostatic electron nonlinear (KEEN) waves, electron plasma waves, Vlasov-Poisson equations, coherent structures in phase space, 
MO3.4

laser-plasma interactions, ponderomotive force, phase space vortices, chaotic dynamics.

\section{INTRODUCTION}

Since the pioneering work of Bernstein, Green and Kruskal on BGK modes in $1957,{ }^{1}$ it has been quite well known that one could play "plasma designer" and find electrostatic fields that are self-consistent with some vortical phase space structure or some manner of trapped particle velocity distribution function (VDF). ${ }^{1-3}$ Of course, the "how do you get there from here?" question had to be gently sidestepped since BGK modes are, strictly

speaking, time independent. This has given rise to quite a persistent trend in rarified theoretical studies ${ }^{4-6}$ where arbitrary changes to the electron or ion velocity distribution functions would be invoked and the troublesome, ubiquitous Landau damping of waves could then be removed with a large nonlinear and arbitrary stroke of the pen. When real laser-plasma interaction experiments ${ }^{7}$ revealed that such structures might conceivably be physically relevant, the premium on self-consistently reaching such states and understanding their origins and limitations became even higher. ${ }^{8}$

We decided to take on this challenge by pursuing the most naïve yet sensible limit imaginable. In this limit we simply consider a 1D homogeneous infinite (periodic boundary conditions) Maxwellian electron plasma, with immobile ions. In it a single prescribed wavelength ponderomotive force is imposed (soon after the start of the simulation), with a short temporal envelope (around a 100 inverse plasma frequencies), and a carrier that has a well defined (high) frequency. We followed the evolution of the plasma after this drive was turned off for another 1000 or more inverse plasma frequencies. For fixed (but many) wavenumber(s), we varied the carrier frequency of the drive and to our surprise, found that first, the response was not single mode ${ }^{8}$ but phase locked and multimode (higher harmonics being indispensable), and second, that not only can electron acoustic type waves (but now multimode) be sustained nonlinearly and self-consistently, (as well as electron plasma waves, of course) and second, that KEEN waves driven by a wide range of frequencies, hitherto thought to be inaccessible for coherent collective wave motion of a plasma (in between EPW and EAW frequencies) were sustainable 
as well. Our studies indicate that this apparent band gap, that is certainly present in linear theory, can be filled with highly nonlinear strictly kinetic (without a fluid limit, never for a Maxwellian) multimode KEEN waves. The ponderomotive driver has to sufficiently perturb the distribution function locally around its phase velocity in order to give the multimode KEEN wave a chance to survive. This increased local slope reduction of the $\mathrm{e}^{-} \mathrm{VDF}$ is a prerequisite for trapped particle modes as is well known. ${ }^{1-8}$ Oddly enough, however, it is at lower drive amplitudes that the response of the plasma is chaotic and insufficiently organizable. These observations bring into question the hope that a perturbative theory which extends (the rather singular yet formally linear) Van Kampen modes ${ }^{4}$ to some nonlinear but manageably small amplitude single mode theory will possibly find use in actual laser-plasma kinetic physics. ${ }^{6,8}$ As the drive amplitude is increased, the collective coherent multimode response of KEEN waves becomes possible and eventually takes root enough to sustain itself indefinitely (within the context of the V-P 1D description). Furthermore, and most gratifyingly, the sequential introduction of multiple KEEN drives or of KEEN and EPW drives does not destroy the waves in our simulations. KEEN waves persist in both kinds of "impure" cases, giving more credence to their inherent stability and potential universality. We have found that any frequency within at least $50 \%$ on either side of the EAW frequency can be used to drive KEENs. Besides, for all these frequencies and even at the EAW frequency, what results is a phase locked multimode wave whose second harmonic is twice as large as its fundamental, its third harmonic, just as large as the fundamental and the forth harmonic, only half as large as the fundamental. These phase locked multimode aspects are entirely absent in any EAW prescription of the past. ${ }^{5-8}$ In contrast to KEEN waves, the tuning width of EPWs is of order one or two percent or less, typically. This means that the band of frequencies within which KEEN waves may be excited is very wide and certainly not the postulated single frequency, single mode, EAW response, or the well known EPW response, ${ }^{2-4}$ but something else entirely.

\section{FORMULATION OF THE PROBLEM}

We solve the Vlasov-Poisson set of equations in a uniform plasma with periodic boundary conditions assuming an initially Maxwellian velocity 
distribution function of width one in natural units. ${ }^{1-4}$ The method of solution is the split operator semi-Lagrangian cubic spline interpolation scheme which is quite popular since the pioneering work of Chang and Knorr. ${ }^{10}$ We introduce an externally imposed ponderomotive (electrostatic) force driver (PFD) which may be thought of as being due to the beating of two counter-propagating electromagnetic waves. ${ }^{9}$ The PFD is simply added to the self-consistent electric field term in the Vlasov equation. For a single KEEN wave simulation, the PFD is characterized by an amplitude, $a_{\text {Drive, wavenumber, }}$ $k_{\text {Drive }}$, which must be a multiple of the simulation domain size, a frequency, $\omega_{\text {Drive }}$, and a temporal envelop. We use the difference between two hyperbolic tangent functions to obtain a smoothly varying hat type function with a start up ramp of 10 inverse plasma frequency units, a duration of a hundred and a ramp down of 10 units as well. We have compared ramp down rates of 10, 50 and a 100 so as to detect any peculiarities associated with the fast case. We found our results to scale adiabatically with the change in ramp down rate, which was reassuring. The drive amplitude that enters the Vlasov equation is the spatial derivative of the ponderomotive potential, which itself is simply the product of the oscillatory velocities of electrons in the E field of each of the two crossing lasers ${ }^{9}$ divided by the electron thermal velocity of the plasma squared. By $a_{\text {Drive }}$ we mean this (normalized) velocity squared quantity. We find that interesting and stable behavior is obtained for drive amplitudes above 0.1 . For a clean KEEN wave, 0.2 and above is preferable. For a green pump laser at $2 \times 10^{15} \mathrm{~W} / \mathrm{cm}^{2}$ and an orange colored probe at a tenth that intensity, and a $0.5 \mathrm{keV}$ electron temperature, we find the drive amplitude to be above 0.23 , which qualifies as a good KEEN wave generation drive level. This can easily be achieved on Trident ${ }^{7}$ at LANL with ten times higher intensities being available as well. The resolution used in these simulations is 2048 in space 4096 in velocity for a $\mathrm{v}$ range of -6 to 6 and 4 modes in $\mathrm{x}$. The time resolution was 0.05 in inverse plasma frequency units. This was seen to closely resemble 0.01 resolution runs but be significantly different than 0.1 runs.

\section{VLASOV SIMULATION RESULTS}

We show the late time phase space images of a KEEN wave in Fig. 1. Fig. 2 shows the density response in Fourier space as a function of time. The first case is driven hard enough that it produces a long lasting and undamped 
KEEN wave. This is not so for the third case shown in Fig. 2. The second case is for marginal drive. The long time stability issue as a function of drive amplitude is also addressed in Fig. 3. Here we plot the values of the first 6 modes of $\mathrm{f}$ in $\mathrm{k}$ space, RMS averaged over velocity (in a velocity coordinate which is shifted by the phase velocity of the driver, with $n$ the mode number):

$$
\left\langle\hat{f}^{(n)}\right\rangle(t)=\sqrt{\left\{\int_{-\bar{v}_{\text {Max }}}^{\bar{v}_{\max }}\left|\hat{f}^{(n)}(\overline{\mathrm{v}}, t)\right|^{2} d \overline{\mathrm{v}}\right\}} \cdot
$$

We can thus determine which harmonics survive and are essential pieces of a KEEN wave, both from the average over $\mathrm{v}$ of each $\mathrm{f}$ mode, whose magnitudes are the rho's plotted in Fig. 2, and by the velocity space average of each f mode's magnitude squared, as shown in Fig. 3.

Fig. 4 shows the changes that occur to the zero order $\mathrm{e}^{-}$VDF when the KEEN wave is formed. The anticipated reduction of the slope of the distribution function is seen to arise in these self-consistently generated waves only if the drive amplitude is high enough. Fig. 5 shows the reconstruction of $\mathrm{f}$ $-\mathrm{f}_{0}$ from $0,1,2,3$ and 4 first spatial modes that gets very close to the full KEEN wave in this 0.2 drive amplitude case. The last frame is the very small difference between the "up to the $4^{\text {th }}$ harmonic approximation" reconstruction and the full solution. Fig 6 shows this more clearly still with the absolute values of the space-time Fourier transformed (fast carriers surgically removed) components of $\mathrm{f}$ being plotted as a function of $\mathrm{v}-\mathrm{v}_{\phi}$. The "few significant coupled harmonics" nature of a KEEN wave is apparent. These harmonics are phase locked, as one may expect from their coherent multimode dynamical orbits in phase space. ${ }^{11}$ The initial $\mathrm{e}^{-}$VDF need not be Maxwellian for a KEEN wave to form. In fact, when a weak amplitude drive is applied but the initial $\mathrm{e}^{-} \mathrm{VDF}$ is extracted from a stronger drive asymptotic state, KEEN waves are found to arise and persist even for that low drive amplitude. The surprise is that this degree of self-organization is apparently possible in a very high dimensional dynamical system (or field theory) well beyond the few coupled oscillator limit where nonlinear stability is more commonly achieved. We are building a parallel version of the Vlasov-Poisson code so as to explore more efficiently these newly found coherent multimode nonlinear kinetic waves in the traditional "band gap" which was thought to exist in plasma physics and laser-plasma interactions. 


\section{ACKNOWLEDGMENTS}

This work was supported by the DOE Grant DE-FG03-03NA00059. We would like to thank D. S. Montgomery for his pioneering and inspirational experiments which gave us the impetus to tackle the EAW existence problem with fully nonlinear, self-consistent and untruncated Vlasov-Poisson simulations. We also would like to thank H. Rose, S. Brunner, E. Williams, W. Manheimer, and W. Kruer for valuable discussions and encouragement.

\section{REFERENCES}

1. I. Bernstein et al., Phys. Rev. 108, 546, (1957).

2. R. C. Davidson, Methods in Nonlinear Plasma Theory, Academic Press, 1972.

3. R. Z. Sagdeev and A. A. Galeev, Nonlinear Plasma Theory, W. A. Benjamin, Inc., 1969.

4. N. G. Van Kampen and B. U. Felderhof, Theoretical Methods in Plasma Physics, North-Holland, 1967.

5. H. Schamel, Phys. Plasmas 7, 4831, (2000) and references therein.

6. J. P. Holloway and J. J. Dorning, Phys. Rev. A44, 3856, (1991), M. Buchanan and J. Dorning, Phys. Rev. E50, 1465, (1994), E52, 3015 (1995), C. Lancellotti and J. J. Dorning, Phys. Rev. Lett. 81, 5137, 1998 and references therein.

7. D. S. Montgomery et al., Phys. Rev. Lett. 87, 155001, (2001).

8. H. Rose and D. Russell, Phys. Plasmas 8, 4784, (2001).

9. W. L. Kruer, The Physics of Laser Plasma Interactions, Addison-Wesley, 1988.

10. A. Ghizzo, et al., J. Comp. Phys., 90, 431 (1990)

11. B. Afeyan et al., submitted to Phys. Rev. Lett., 2003. 


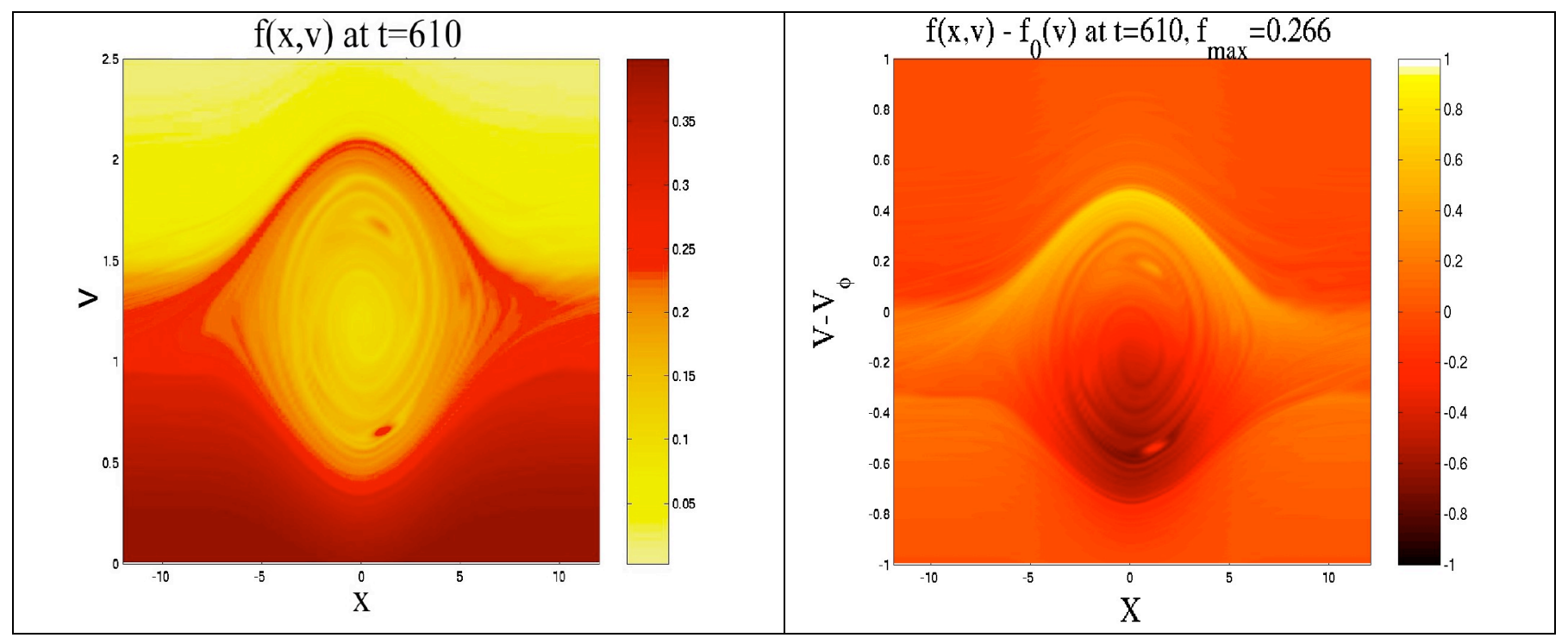

Figure 1. Late time phase space snapshots of a KEEN wave ponderomotively driven with an amplitude $0.4, k_{\text {Drive }}=0.26$ and $\omega_{\text {Drive }}=0.37$. Also shown is a phase space snapshot where the velocity axis is shifted to be centered with respect to the phase velocity of the driver and where the initial Maxwellian VDF has been subtracted. Of the four vortices that exist in the computational domain, we show just one for clarity.

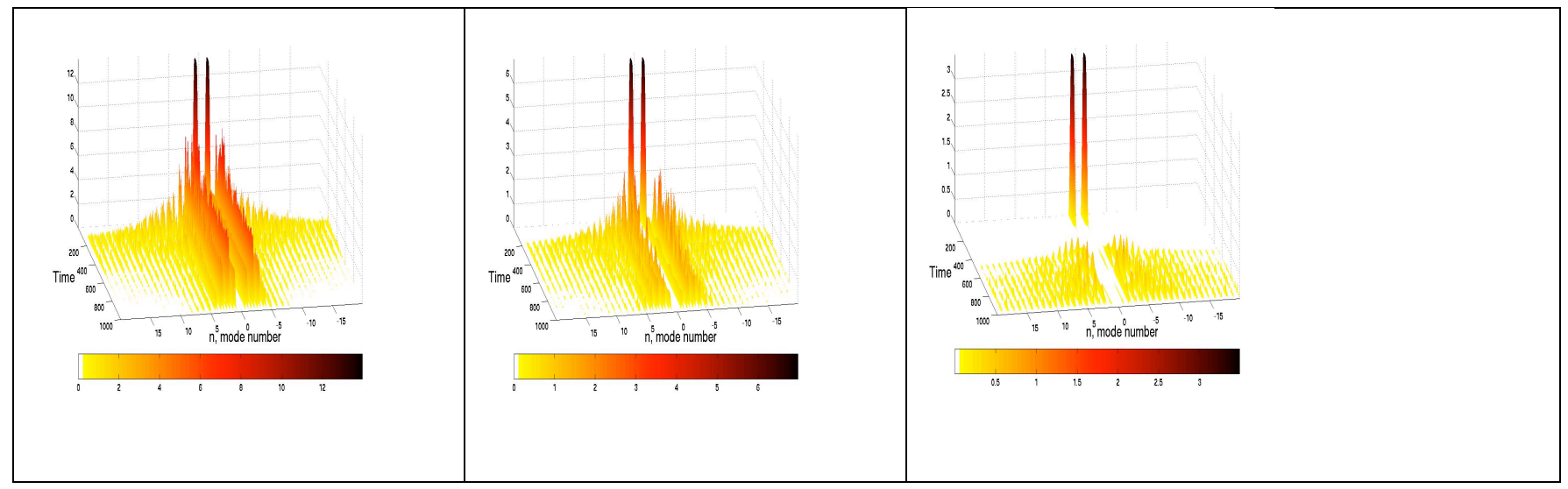

Figure 2. The absolute value of the density response as a function of mode number $\mathrm{k} / \mathrm{k}_{\text {Drive }}$ and time. Three amplitude drive cases are shown side by side. $\mathrm{a}_{\text {Drive }}=0.2,0.1$ and 0.05 . The second is a marginal KEEN wave producing case and the third is an abortive one. Note that around 20 modes are involved 
with the initial creation process (in the first two cases where KEEN waves can be formed at all) but 4 survive with any appreciable amplitude in steady state. $\omega_{\text {Drive }}=0.37$ and $k_{\text {Drive }}=0.26$ in all three cases. 
Figure 3. The RMS velocity averaged values of the first few $(0,1, \ldots, 6) \mathrm{k}$ space modes of KEEN waves driven at amplitudes $0.3,0.2,0.1$ and 0.05 . Note that at 0.1 and 0.05 , the long term trend of the RMS values of f decay while at higher drive amplitudes the time asymptotic values are flat. The higher the drive amplitude, the smoother the RMS response becomes. The multi-mode self-organization is faster and

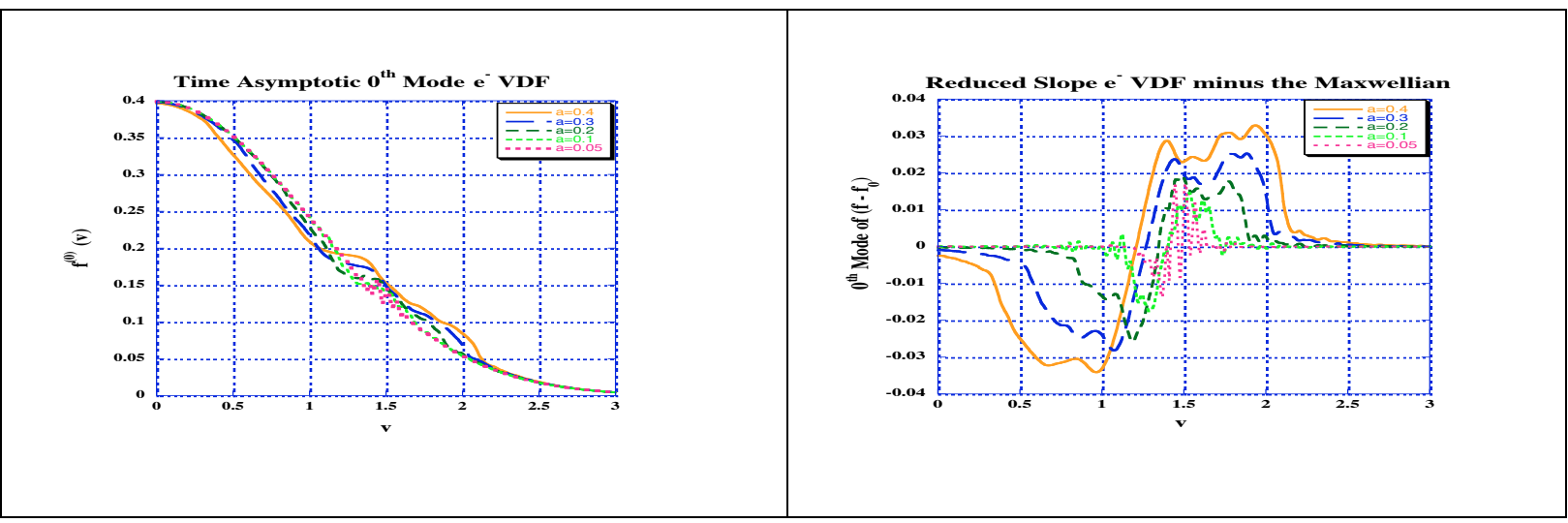

more efficient with higher drive amplitudes.

Figure 4. The drive amplitude dependence of the time asymptotic form of the non-oscillatory component of the $\mathrm{e}^{-} \mathrm{VDF}$ of a KEEN wave, $\mathrm{f}^{(0)}$, as well as $\mathrm{f}^{(0)}-\mathrm{f}_{0}$, where $\mathrm{f}_{0}$ is the initial Maxwellian. Drive amplitudes of $0.4,0.3,0.2$, 0.1 and 0.05 are shown.

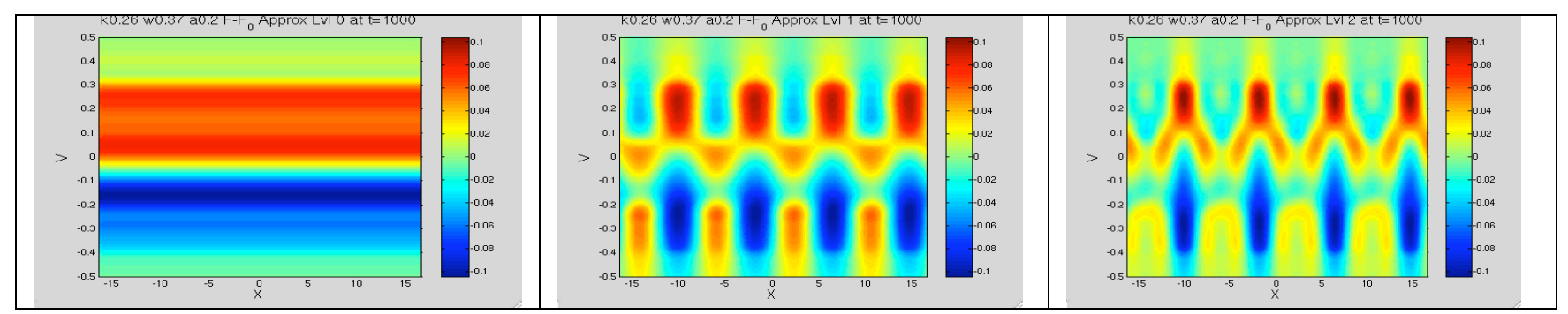




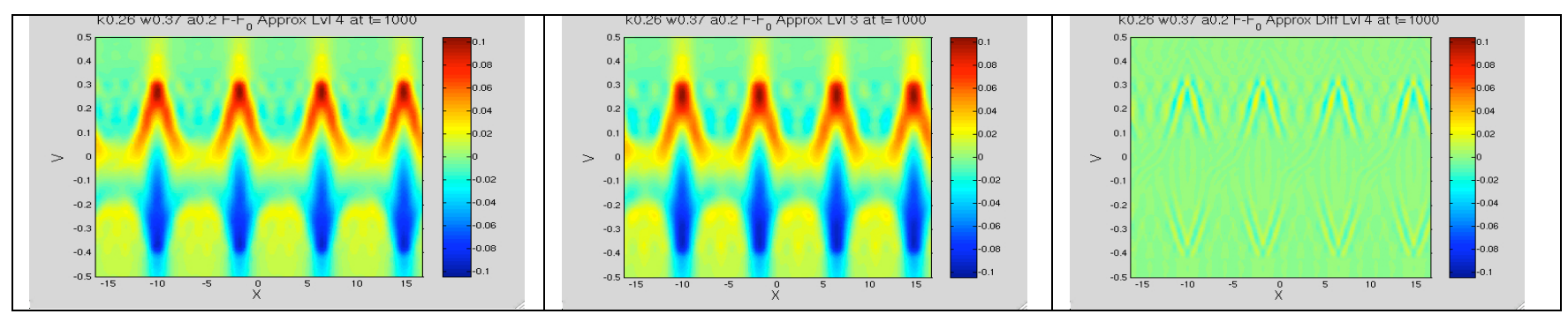

Figure 5. Partial mode reconstructions of a large amplitude drive $\left(\mathrm{a}_{\text {Drive }}=0.4\right)$ KEEN wave. Note that very small corrections are included in modes higher than four.
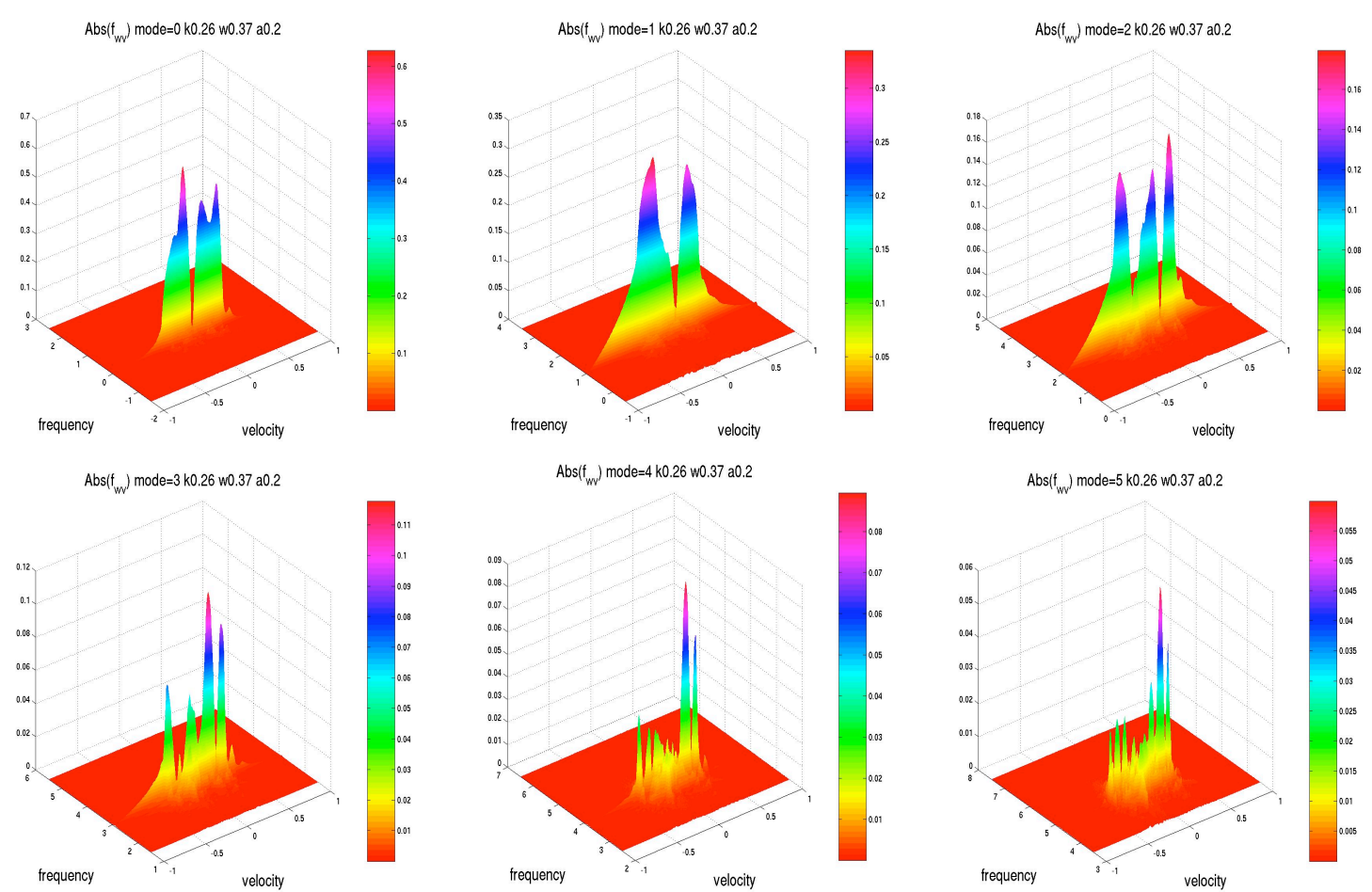

Fig 6. The absolute values of the zeroth and the next 5 spatial modes of $f$ vs frequency (normalized to the drive frequency) and velocity (in phase velocity shifted coordinates). This is a drive amplitude $0.2 \mathrm{KEEN}$ wave whose drive frequency was 0.37 and wavenumber, 0.26 . Note the very pure harmonics in frequency in the lowest order modes, becoming more and more extended in frequency content as we look at higher and higher modes. 


\title{
Optical Mixing Controlled Stimulated Scattering instabilities: Suppression of SRS by the Controlled Introduction of Ion Acoustic and Electron Plasma Wave Turbulence
}

\author{
Bedros Afeyan, ${ }^{a}$ M. Mardirian, ${ }^{a}$ K. Won, ${ }^{a}$ D. S. Montgomery, ${ }^{b}$ J. Hammer, ${ }^{c}$ R. \\ K. Kirkwood, ${ }^{c}$ A. J. Schmitt ${ }^{d}$ \\ ${ }^{a}$ Polymath Research Inc., Pleasanton, CA \\ ${ }^{b}$ Los Alamos National Laboratory, Los Alamos, NM \\ ${ }^{\mathrm{c}}$ Lawrence Livermore National Laboratory, Livermore, CA \\ ${ }^{d}$ Naval Research Laboratories, Washington, DC
}

\begin{abstract}
In a series of experiments on the Omega laser facility at LLE, we have demonstrated the suppression of SRS in prescribed spectral windows due to the presence of externally controlled levels of ion acoustic waves (IAW, by crossing two blue beams at the Mach -1 surface) and electron plasma waves (EPW, by crossing a blue and a green beam around a tenth critical density plasma) generated via optical mixing. We have further observed SRS backscattering of a green beam when crossed with a blue pump beam, in whose absence, that (green beam) backscattering signature was five times smaller. This is direct evidence for green beam amplification when crossed with the blue. Additional proof comes from transmitted green beam measurements. A combination of these techniques may allow the suppression of unacceptable levels of SRS near the light entrance hole of large-scale hohlraums on the NIF or LMJ.
\end{abstract}

Keywords: Optical mixing generation, crossing laser beams, SRS, SBS, parametric instabilities, controlling laser-plasma interactions, plasma fluctuations and turbulence, laser beam filamentation 


\section{INTRODUCTION}

The success of laser based inertial confinement fusion (ICF) depends critically on the ability to convert the incident laser energy to $\mathrm{X}$ rays at the walls of a hohlraum, in the case of indirect drive, ${ }^{1}$ and to plasma thermal energy so as to drive an ablation front, in the case of direct drive. ${ }^{2}$ Parametric instabilities ${ }^{3}$ inevitably occur when high enough intensity $\left(\mathrm{I}_{\text {laser }} \sim 10^{15} \mathrm{~W} / \mathrm{cm}^{2}\right)$ coherent laser radiation is introduced into a low enough temperature $\left(\mathrm{T}_{\mathrm{e}}<5\right.$ $\mathrm{keV})$, and high enough density $\left(\mathrm{n}_{\mathrm{e}} / \mathrm{n}_{\mathrm{c}}>.05\right)$ and long enough scale length $\left(\mathrm{L}_{\mathrm{n}}\right.$ $>100$ s of $\mu \mathrm{ms}$ ) plasmas. Such coherent wave-wave interactions, ${ }^{3}$ for instance, SRS, SBS and filamentation, must be mitigated so that an inadmissibly high fraction of the incident laser energy is not misdirected via nonlinear scattering losses. For the success of ICF, excessive hot electron preheat can not be tolerated either, but which may, nevertheless, occur with SRS and $2 \omega_{\mathrm{pe}}{ }^{1-3}$

For the last four years, on Omega, at LLE, as part of the NLUF program, we have studied ways to mitigate the severity of these processes. Our idea was to use crossing laser beams in order to introduce controlled levels of plasma fluctuations and turbulence so as to make the plasma inhospitable to coherent, highly correlated three wave interactions. We have already shown ${ }^{4}$ that at the Mach -1 surface, when two blue beams cross and generate a large amplitude IAW, they strongly diminish the SRBS of the blue beam coming from the vicinity of that density. This is shown in Fig. 1 both for weak and strong IAW damping cases. We can see from Fig. 1a that the reduction of the reflectivity of SRS (in the proper wavelength window corresponding to the vicinity of the Mach -1 surface) in the presence of a large amplitude IAW (driven by two high intensity lasers) is by a factor of 3-5 when compared to the SRS levels generated when the beam crossing the pump beam is much weaker $\left(1 / 15^{\text {th }}\right.$ the intensity of the pump) and hence unable to create large amplitude IAWs. Even more so, in the case of weak IAW damping, (where Be targets were used which do not have Hydrogen in them as do the $\mathrm{CH}$ targets) the effect was a factor of 7 to 8 SRS reflectivity reduction from the proper wavelength window when comparing 1:1 to $1 / 10: 1$ intensity ratio cases between probe and pump laser beams, respectively. Fig. 2 shows SRS suppression by a factor 2-3 when large amplitude EPWs are optically generated and act as hindrances to a witness beam's SRBS process. 
More recently, we have conducted experiments where we crossed high intensity blue and green beams that could, around a tenth critical density (for the blue), resonantly drive an EPW. Fig. 3 shows the experimental configuration for these experiments. We have various means of diagnosing the resonant interactions between crossing blue and green beams. One novel way is via the observation of green beam SRBS with spectral signatures far weaker when the crossing blue beam is not present. We interpret this new result as the amplification of the Green beam via optical mixing with the blue pump beam and the subsequent Raman backscattering of the green beam from the peak of the exploding foil density profile when its intensity is boosted so as to become high enough to produce significant levels of SRBS. This is quite interesting since it shows that energy transfer between crossing beams leaves the amplified beam coherent enough to be able to instigate high levels of backscattering of its own.

In our next set of experiments, we hope to study these phenomena more carefully and to combine these processes to see just how far we can go to suppress parametric instabilities in fluctuating and turbulent plasmas without inadvertently triggering some other undesirable process.

\section{EXPERIMENTAL RESULTS}

We have shot 7-10 $\mu \mathrm{m} \mathrm{CH}$ exploding foils as well as $5 \mu \mathrm{m}$ Be foils to create sub-tenth-critical plasmas with parabolic density profiles. In our most recent experiments, with crossing blue and green laser beams, four $500 \mathrm{~J}$ beams per side were used for $1 \mathrm{~ns}$ to create the exploding foil plasma and four more per side to heat it for another ns. In the middle of the second set of heater beams, the interaction beams were introduced.

Fig. 2 shows the suppression of SRS of a witness beam (BL30) in the presence of crossing blue (BL65) and green beams, which resonantly generate an EPW as the appropriate (roughly a ninth critical density for the blue) density is reached at the peak of the exploding foil plasma.

Fig. 3 shows the configuration of the experiment when BL60 as opposed to 65 was used as the blue pump beam. The Green beam TBD introduced an occlusion in front of BL65. We observed enhanced green beam amplification 
due to the presence of the blue pump beam. This is ascertained in Fig. 4 by the novel and unexpected measurement of significant green beam Raman backscatter enhancement (by a factor of 15) triggered directly by the green beam being crossed with a blue beam.

\section{ACKNOWLEDGMENTS}

This work was supported by DOE Grants DE-FG03-03SF22690 and DEFG03-03NA00059. We would like to thank the Omega crew at LLE as well as J. Soures, R. Bahr, S. Morse, G. Pien and M. Bonino, for their invaluable support and O. Landen and LLNL for providing a wider spot DPP and a TBD diagnostic for the green beam which made the new results reported here possible.

\section{REFERENCES}

12. J. D. Lindl, Inertial Confinement Fusion, AIP Press, 1998.

13. S. E. Bodner, et al., Phys. Plasmas, 5, 1901, 1998.

14. W. L. Kruer, The Physics of Laser Plasma Interactions, Addison-Wesley, 1988.

15. B. Afeyan, et al., IFSA Proceedings, Elsevier, p331, 1999. 


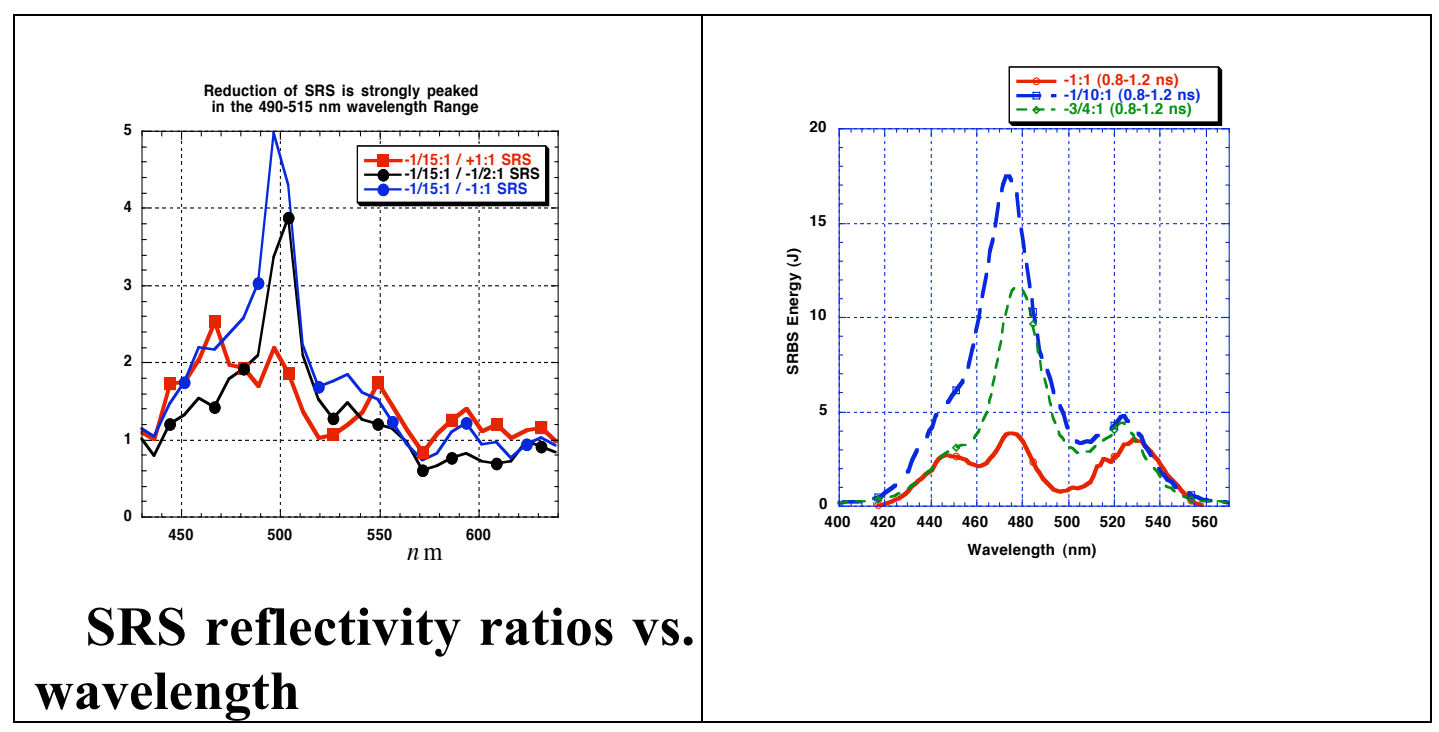

Figure 1. Reduction of Raman backscattering levels in the presence of two high intensity crossed blue beams compared to scattering levels when the probe beam is weak in a) $10 \mu \mathrm{m} \mathrm{CH}$ targets which correspond to high IAW damping (reduction is a factor of 5 in the wavelength window around the Mach -1 surface where resonant interaction is possible) and b) $5 \mu \mathrm{m}$ Be targets which correspond to the weak IAW damping regime with equivalent hydrodynamic expansion properties.
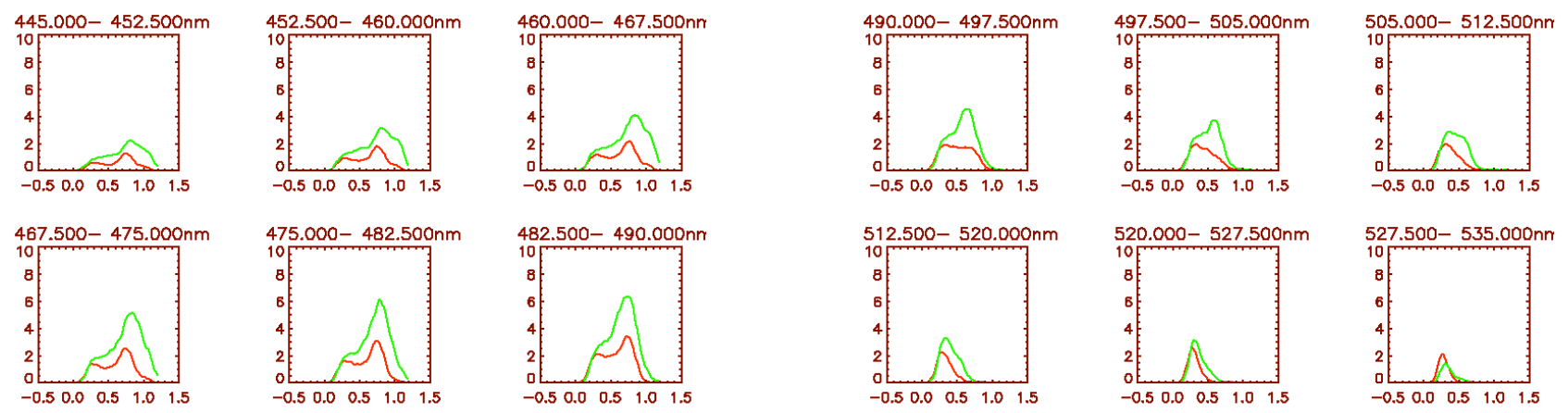
Fig 2. Plotted is Raman backscatter power vs. time in contiguous wavelength bins. This is wavelength partitioning of the witness beam's SRBS when a pump and probe beam pair cross to resonantly generate an EPW (Red) and when the blue pump beam is turned off (Green). We find factors of 3 reduction in SRBS in the presence of an externally generated EPW in a wavelength bin and time window which is consistent with the peak of the plasma density profile being at the right density where the EPW could have been driven by crossing blue and green beams, as inferred from LASNEX simulations. Green beam energy was 100J (probe) and the blue beams (pump and witness) were at $500 \mathrm{~J}$.

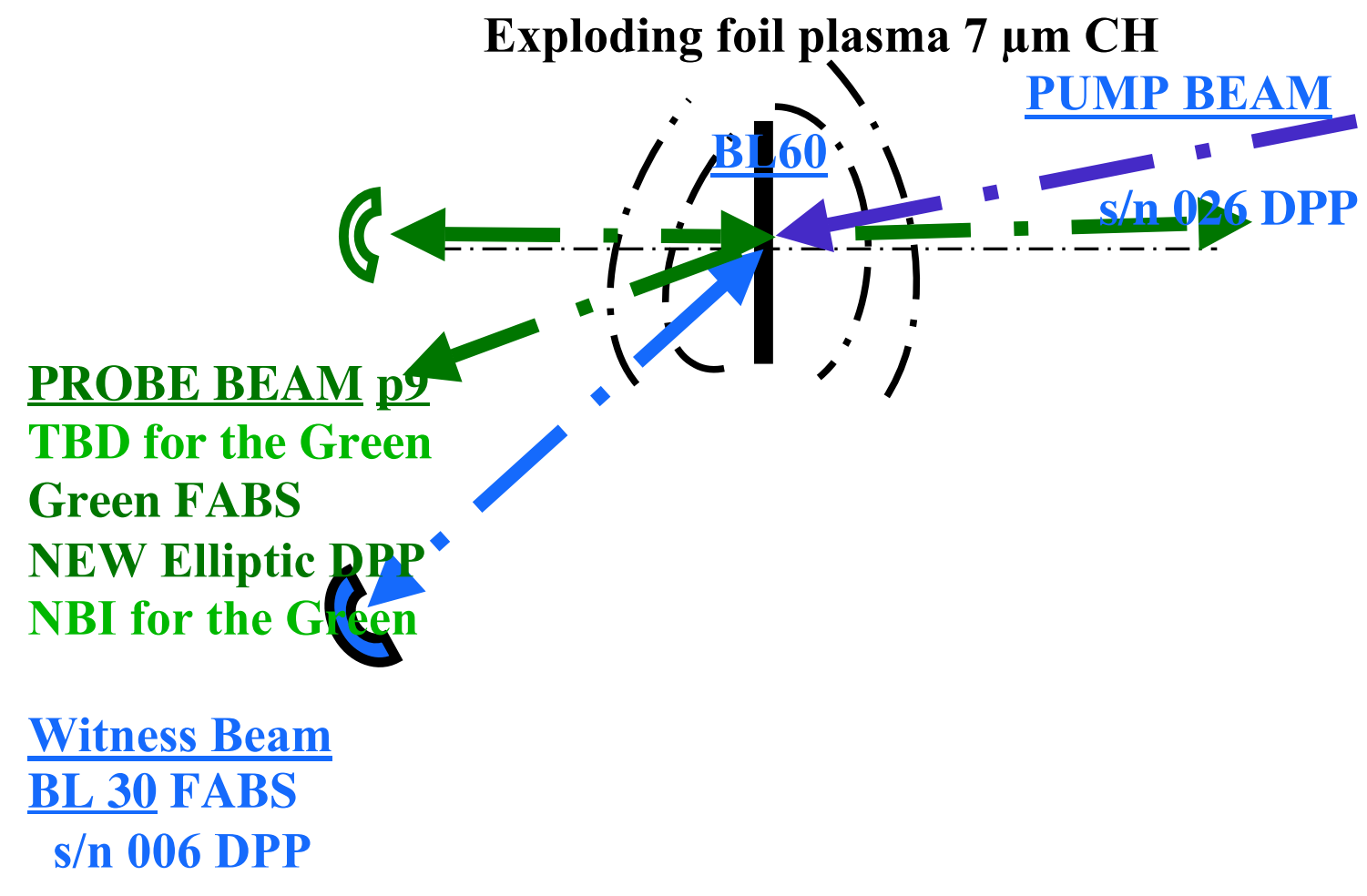

Figure 3. This is a schematic diagram of the experimental configuration of the three interaction beams used (crossing pumpBL60, probe-green, and witness-BL30 beams) and the optical 
diagnostics fielded. Full aperture backscatter stations (FABS) for SRS and SBS were used on the blue witness beam BL30 and the green probe beam. Near backscatter imaging (NBI) on BL30 and the green beam as well as a transmission beam diagnostic (TBD) for the green beam alone were also deployed. All three interaction beams had distributed phase plates or DPPs. BL60 is the pump beam which amplifies the green probe and resonantly excites an EPW via optical mixing with the green. Its backscatter is not measured due to space and other material resource constraints in the target chamber on Omega at LLE.

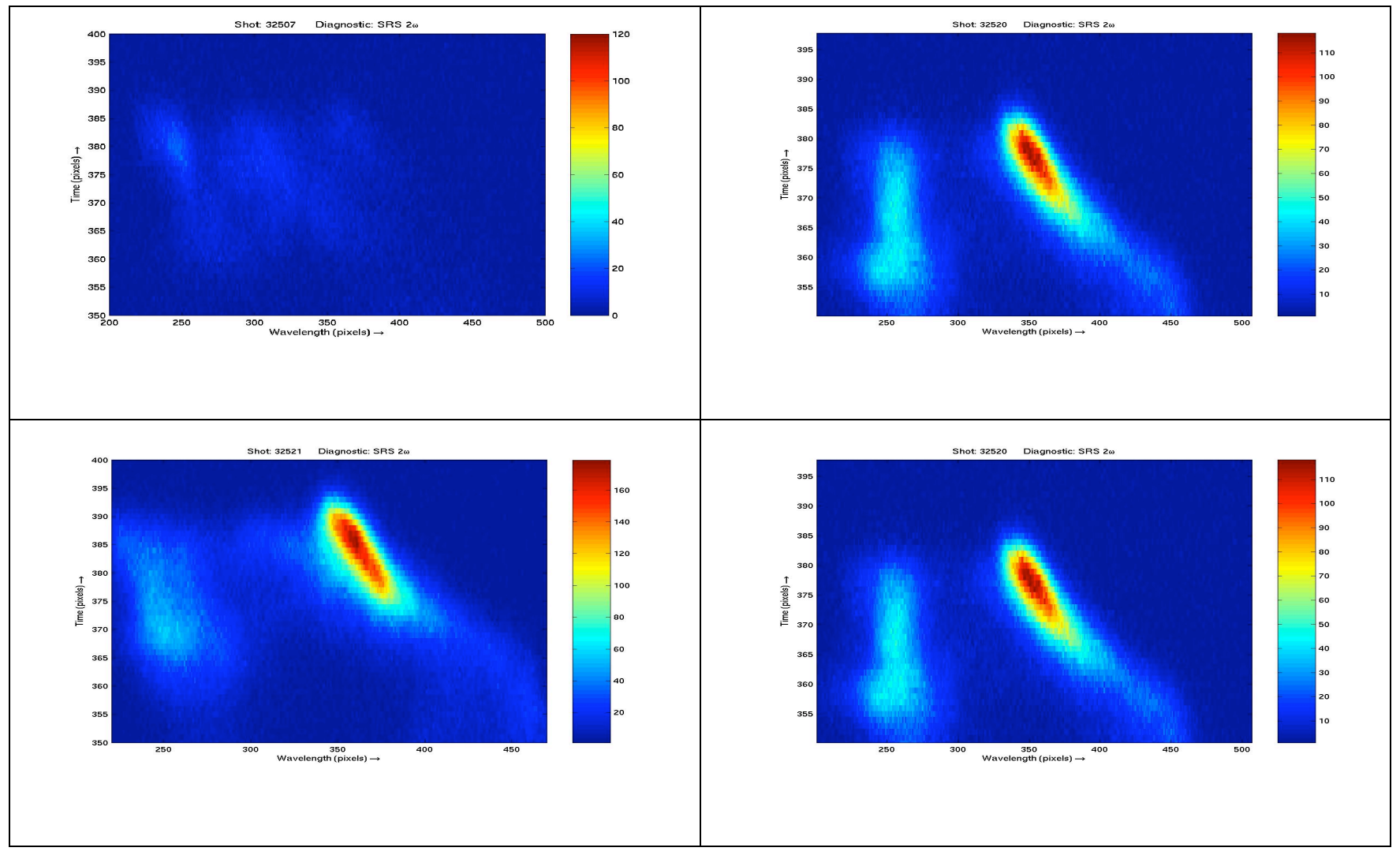




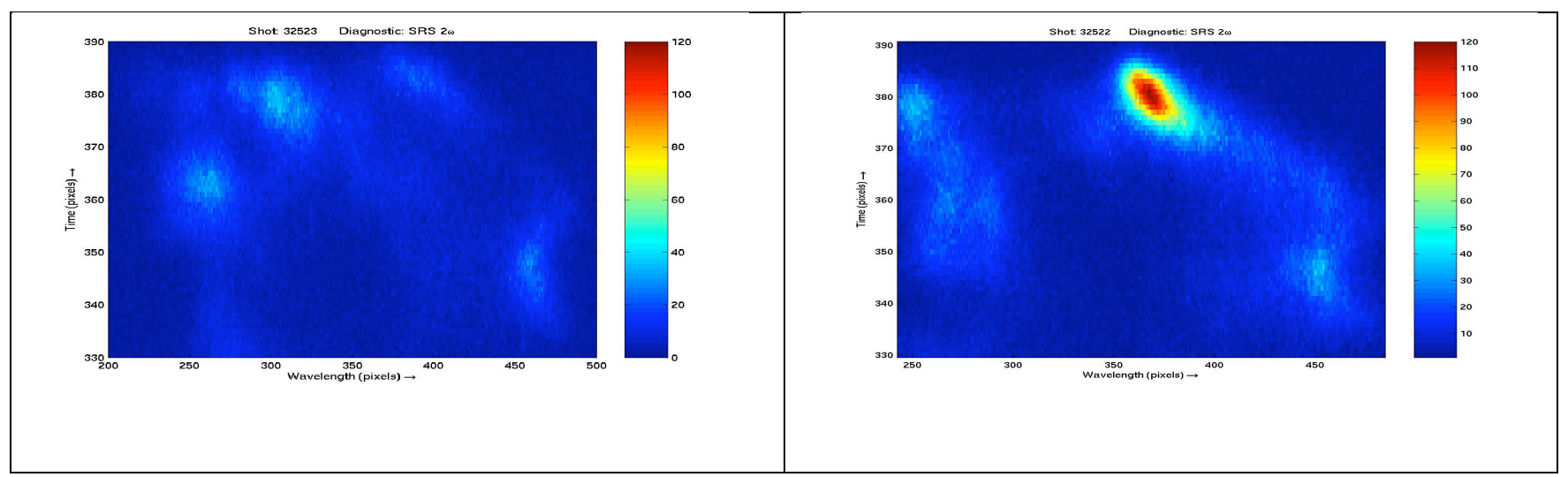

Figure 4. Green beam SRS backscattering comparisons. Optical mixing amplified green beam Raman backscattering (SRBS) is observed when the incident green beam energy is 94J. At this energy, green beam SRBS is very weak. However, when a large amplitude blue pump beam is also present crossing the green from the opposite side, it amplifies the green beam to such as extent (around a factor of two) that now the green beam's SRS backscatter is fifteen times higher in peak magnitude. The second row compares cases with two blue pump beam energies and shows similar results with enhanced filamentation signature SRS coming from very low density plasma when the blue pump beam is on. The third row is similar to the first except the hydrodynamics of the target was changed due to two heater beams being dropped in order to accommodate the green beam TBD diagnostic. This made the density of the plasma $25 \%$ higher at corresponding times during the pulse as well as the temperature lower, which promoted larger levels of filamentation. Despite all that, remarkably similar backscatter enhancement is seen in the presence of the Blue crossing beam. The hydro was calculated using LASNEX. Row 1: $\mathrm{E}_{\mathrm{G}}=94 \mathrm{~J}, \mathrm{E}_{\mathrm{BL} 60}=0$ and $489 \mathrm{~J}$. Row $2: \mathrm{E}_{\mathrm{G}}=94 \mathrm{~J}, \mathrm{E}_{\mathrm{BL} 60}=374 \mathrm{~J}$ and $489 \mathrm{~J}$. Row 3: $\mathrm{E}_{\mathrm{G}}=94 \mathrm{~J}, \mathrm{E}_{\mathrm{BL} 60}=0$ and $485 \mathrm{~J}$. 